\title{
A Study of the Dirichlet Priors for Term Frequency Normalisation
}

\author{
Ben $\mathrm{He}$ \\ Department of Computing Science \\ University of Glasgow \\ Glasgow, United Kingdom \\ ben@dcs.gla.ac.uk
}

\author{
ladh Ounis \\ Department of Computing Science \\ University of Glasgow \\ Glasgow, United Kingdom \\ ounis@dcs.gla.ac.uk
}

\begin{abstract}
In Information Retrieval (IR), the Dirichlet Priors have been applied to the smoothing technique of the language modeling approach. In this paper, we apply the Dirichlet Priors to the term frequency normalisation of the classical BM25 probabilistic model and the Divergence from Randomness PL2 model. The contributions of this paper are twofold. First, through extensive experiments on four TREC collections, we show that the newly generated models, to which the Dirichlet Priors normalisation is applied, provide robust and effective performance. Second, we propose a novel theoreticallydriven approach to the automatic parameter tuning of the Dirichlet Priors normalisation. Experiments show that this tuning approach optimises the retrieval performance of the newly generated Dirichlet Priors-based weighting models.
\end{abstract}

\section{Categories and Subject Descriptors}

H.3.3 [Information Storage and Retrieval]: Retrieval models

\section{General Terms}

Experimentation, Performance, Theory

\section{Keywords}

Term frequency normalisation, weighting model, Dirichlet Priors

\section{INTRODUCTION}

Document ranking is a crucial issue in Information Retrieval (IR), which is usually based on a weighting model [9]. Almost all weighting models take term frequency $(t f)$, the number of occurrences of a query term in a document, into consideration as a basic factor for document ranking.

However, the term frequency is dependent on the document length, i.e. the number of tokens in a document.

Permission to make digital or hard copies of all or part of this work for personal or classroom use is granted without fee provided that copies are not made or distributed for profit or commercial advantage and that copies bear this notice and the full citation on the first page. To copy otherwise, to republish, to post on servers or to redistribute to lists, requires prior specific permission and/or a fee.

SIGIR'05, August 15-19, 2005, Salvador, Brazil.

Copyright 2005 ACM 1-59593-034-5/05/0008 ...\$5.00.
In [11], Singhal et al. summarised the following two aspects of the effect of document length on $t f$ :

- The same term usually occurs repeatedly in long documents.

- A long document has usually a large size of vocabulary.

As a consequence, $t f$ needs to be normalised by using a technique called term frequency normalisation.

Many normalisation methods have been developed in the past, including the normalisation 2 [1] and BM25's normalisation component [10]. Moreover, as suggested in [1], the Dirichlet Priors, which have been applied to the IR language modeling approach [13], can also be applied to the $t f$ normalisation. We will introduce these normalisation methods in the next section. In this paper, we denote the $t f$ normalisation using the Dirichlet Priors as the Dirichlet Priors normalisation, and denote the weighting model, to which the Dirichlet Priors normalisation is applied, as the Dirichlet Priors-based model.

In this paper, we study the application of the Dirichlet Priors normalisation on a representative of two families of weighting models, including the classical BM25 probabilistic model [10] and the Divergence from Randomness (DFR) PL2 model [1]. In our extensive experiments, we show that the Dirichlet priors-based models achieve robust and effective retrieval performance over diverse TREC collections. Experiments also show that there is a justifiable need for the tuning of the parameter of the Dirichlet Priors normalisation. In particular, we propose a novel automatic theoretically-driven tuning methodology for the Dirichlet Priors normalisation. As mentioned above, there is a dependence between $t f$ and the document length. Therefore, the purpose of the $t f$ normalisation is to adjust the dependence between the normalised term frequency and the document length. In our tuning method, this dependence is interpreted as the correlation between the two variables. In our experiments, we show that the optimal parameter values, which give the best mean average precision, result in stable correlation measures. In particular, our tuning approach seems to be both collection-independent and query-independent.

The remainder of this paper is organised as follows. In Section 2, we introduce some related work, including the above mentioned normalisation methods and the weighting models to which these normalisation methods are applied. We apply the Dirichlet Priors normalisation to BM25 and PL2 in Section 3 and describe the proposed methodology for tuning the Dirichlet Priors normalisation in Section 4. 
In Sections 5 and 6, we present our experimental setting and evaluation results. Finally, we conclude the work and suggest future directions in Section 7.

\section{RELATED WORK}

As one of the most established weighting models, BM25 computes the relevance score of a document $d$ for a query $Q$ by the following formula:

$$
\operatorname{score}(d, Q)=\sum_{t \in Q} w^{(1)} \frac{\left(k_{1}+1\right) t f}{K+t f} \frac{\left(k_{3}+1\right) q t f}{k_{3}+q t f}
$$

where $q t f$ is the query term frequency; $w^{(1)}$ is the $i d f$ factor, which is given by:

$$
w^{(1)}=\log _{2} \frac{N-N_{t}+0.5}{N_{t}+0.5}
$$

$N$ is the number of documents in the whole collection. $N_{t}$ is the document frequency of term $t$.

$K$ is:

$$
k_{1}\left((1-b)+b \frac{l}{a v g_{-} l}\right)
$$

$l$ and $a v g \_l$ are the document length and the average document length in the collection, respectively. The document length refers to the number of tokens in a document. $k_{1}$, $k_{3}$ and $b$ are parameters. The default setting is $k_{1}=1.2$, $k_{3}=1000$ and $b=0.75$ [10]. qtf is the number of occurrences of a given term in the query; $t f$ is the within document frequency of the given term.

In the following derivation, we show how the BM25 model implicitly employs a $t f$ normalisation component.

Let $t f n=\frac{t f}{(1-b)+b \cdot \frac{l}{a v g_{-l}}}$, where $t f n$ denotes the normalised term frequency, we obtain:

$$
\begin{aligned}
\operatorname{score}(d, Q) & =\sum_{t \in Q} w^{(1)} \frac{k_{1}+1}{\frac{k_{1}}{t f n}+1} \frac{\left(k_{3}+1\right) q t f}{k_{3}+q t f} \\
& =\sum_{t \in Q} w^{(1)} \frac{\left(k_{1}+1\right) t f n}{k_{1}+t f n} \frac{\left(k_{3}+1\right) q t f}{k_{3}+q t f}
\end{aligned}
$$

Hence the term frequency normalisation component of the BM25 formula can be seen as:

$$
t f n=\frac{t f}{(1-b)+b \cdot \frac{l}{a v g_{-} l}}
$$

The above BM25's normalisation component can be seen as a generalisation of Singhal et al.'s pivoted normalisation for normalising the $t f \cdot i d f$ weight [11].

PL2 is one of the divergence from randomness (DFR) document weighting models [2]. Using the PL2 model, the relevance score of a document $d$ for a query $Q$ is given by:

$$
\begin{aligned}
\operatorname{score}(d, Q)= & \sum_{t \in Q} q t f \cdot \frac{1}{t f n+1}\left(t f n \cdot \log _{2} \frac{t f n}{\lambda}\right. \\
& \left.+(\lambda-t f n) \cdot \log _{2} e+0.5 \cdot \log _{2}(2 \pi \cdot t f n)\right)
\end{aligned}
$$

where $\lambda$ is the mean and variance of a Poisson distribution. $q t f$ is the query term frequency.

The normalised term frequency $t f n$ is given by the socalled normalisation $2[1,7]$ :

$$
t f n=t f \cdot \log _{2}\left(1+c \cdot \frac{a v g_{-} l}{l}\right),(c>0)
$$

where $l$ is the document length and $a v g_{-} l$ is the average document length in the whole collection. $t f$ is the original within document term frequency. $c$ is the free parameter of the normalisation method.

The Dirichlet Priors stand for the priors in a Dirichlet distribution, which is a generalisation of the Beta distribution in a multinomial case. In [13], Zhai \& Lafferty have applied the Dirichlet priors in defining their language model for IR. It was also suggested by Amati that the Dirichlet Priors can be used for the $t f$ normalisation [1]:

$$
t f n=\frac{t f+\mu \cdot \frac{t f_{c}}{l_{c}}}{l+\mu} \cdot \mu
$$

where $t f n$ is the normalised term frequency. $l$ is the document length. $t f_{c}$ is the frequency of the given query term in the collection. $l_{c}$ is the number of tokens in the whole collection. $\mu$ is the parameter of the Dirichlet Priors normalisation.

\section{APPLICATIONS OF THE DIRICHLET PRIORS NORMALISATION}

In this section, we apply the Dirichlet Priors normalisation to both PL2 (see Equation (4)) and BM25 (see Equation (1)).

As shown in the previous section, both BM25 and PL2 weighting models employ a $t f$ normalisation component. By replacing the $t f$ normalisation components of the two models with the Dirichlet Priors normalisation, we generate the following new models:

- The Dirichlet Priors-based BM25 model (BM3) is given as our derivation of BM25 in Equation (2), where the normalised term frequency $t f n$ is given by the Dirichlet Priors normalisation in Equation (6). In the rest of this paper, we denote the Dirichlet Priors-based BM25 as BM3 ${ }^{1}$.

- The Dirichlet Priors-based PL2 model (PL3) is given as the PL2 model in Equation (4), where the normalised term frequency $t f n$ is given by the Dirichlet Priors normalisation in Equation (6). We denote this new model as PL3.

In Section 6.1, through extensive experiments, we show that the newly generated Dirichlet Priors-based models lead to robust and effective retrieval performance. Experiments also show that the optimal parameter setting of the parameter $\mu$, which gives the best mean average precision, varies on different collections and query types, indicating that there is a need for tuning the parameter. We describe the proposed tuning method in the next section.

\footnotetext{
${ }^{1}$ In [1], Amati denotes the Dirichlet Priors normalisation as the normalisation 3 . In this paper, we follow his notation and denote a Dirichlet Priors-based model M as M3.
} 


\section{PARAMETER TUNING FOR THE DIRICH- LET PRIORS NORMALISATION}

In this section, we propose a novel theoretically-driven methodology for the automatic tuning of the Dirichlet Priors normalisation. Our proposed methodology is based on measuring the correlation (corr) [4] of the normalised term frequency $(t f n)$ with the document length $(l)$ for a given query term, which is given by:

$$
\operatorname{corr}(t f n, l)=\frac{C O V(t f n, l)}{\sigma(t f n) \sigma(l)}
$$

where $C O V$ stands for covariance. $\sigma(l)$ is the standard deviation of the length of the documents containing the given query term. $\sigma(t f n)$ is the standard deviation of the normalised term frequency of the given query term in all the documents containing the term.

As introduced in Section 1, the purpose of the $t f$ normalisation is to smooth the dependence between $t f n$ and document length, which can be represented by the above correlation formula. We believe that the $t f$ normalisation can be seen as the $t f$ density estimation of the document length. On different collections, the ideal $t f$ density function should result in similar correlation of $t f n$ with the document length. Therefore, the underlying hypothesis of our tuning method is the following:

\section{Hypothesis:}

On different collections, the optimal term frequency normalisation parameter setting provides similar average correlation of the normalised term frequency with the document length for a given set of query terms.

In the above hypothesis, the optimal parameter setting refers to the parameter value that provides the highest mean average precision. Based on the hypothesis, the tuning of the Dirichlet Priors normalisation becomes the issue of identifying the parameter setting that gives an optimal average correlation of $t f n$ with $l$ for the given set of query terms. The proposed tuning methodology can be described as follows:

1. On a training collection, we obtain the optimal parameter setting by using relevance assessment, and then compute the corresponding average $\operatorname{corr}(t f n, l)$ value, which is a collection-independent and queryindependent constant, based on our hypothesis.

2. On a given new collection, and for a given new query set, we apply the parameter setting that gives the optimal average $\operatorname{corr}(t f n, l)$ obtained on the training collection.

Note that in the above tuning process, for a given set of query terms and a particular parameter value, the $\operatorname{corr}(t f n, l)$ value is the average correlation measure for the given set of query terms. Ideally, we could tune the parameter setting for each query term, and apply different parameter settings for different query terms. However, as it is very expensive to carry out such a term-based parameter tuning mechanism, in this paper, we rather assume an optimal average correlation measure so that we do the tuning process for a set of
Table 1: The five weighting models involved in our experiments. The last three models are our baselines.

\begin{tabular}{|c|l|c|}
\hline Model & Formula & Normalisation \\
\hline BM3 & Equation (2) & Equation (6) \\
PL3 & Equation (4) & Equation (6) \\
\hline BM25 & Equation (2) & Equation (3) \\
PL2 & Equation (4) & Equation (5) \\
$t f \cdot i d f$ & Equation (8) & implicitly applied \\
\hline
\end{tabular}

query terms in a batch mode, and apply a unique parameter setting for all the terms in the given set of queries.

According to the study by Zhai \& Lafferty [13], the optimal setting of the parameter $\mu$ of the Dirichlet Priors Smoothing changes with different collections and query sets. Based on the above hypothesis, our explanation is that the length distribution of documents containing a given query term varies with the change of data set, including the collection and query set. As a consequence, a particular correlation $\operatorname{corr}(t f n, l)$ value corresponds to different $\mu$ values on different data sets, and therefore results in different optimal parameter values. In our experiments in Section 6.2, we show that our hypothesis holds on diverse TREC collections.

\section{EXPERIMENTAL ENVIRONMENT}

In our experiments, we evaluate the Dirichlet Priors-based models, i.e. BM3 and PL3, on diverse collections using three baselines, which are BM25, PL2 and the classical $t f \cdot i d f$ weighting models. Table 1 lists the five models involved in our experiments.

Our implementation of the $t f \cdot i d f$ baseline weighting model is a combination of the Okapi's tf [10] and Sparck-Jones' $i d f[12]$ :

$\operatorname{score}(d, Q)=\sum_{t \in Q} q t f \cdot \frac{k_{1} t f}{t f+k_{1}\left(1-b+b \frac{l}{l_{\text {coll }}}\right)} \cdot \log _{2}\left(\frac{N}{N_{t}}+1\right)$

where $q t f$ is the query term frequency of $t . \quad N$ is the number of documents in the whole collection and $N_{t}$ is the document frequency of $t$. $l$ and $l_{\text {coll }}$ are the number of tokens in $d$ and in the whole collection, respectively. $k_{1}$ and $b$ are parameters and their default settings are $k_{1}=1.2$ and $b=$ 0.75 , respectively $[10]$.

We experiment on four TREC collections to evaluate the Dirichlet Priors-based models. The four used collections are the disk1\&2, disk4\&5 (minus the Congressional Record on disk4) of the classical TREC collections ${ }^{2}$, and the WT2G [6] and WT10G [5] Web collections. The test queries are TREC topics that are numbered from 51 to 200 for the disk $1 \& 2$, from 301 to 450 and from $601-700$ for the disk4\&5, from 401 to 450 for the WT2G, and from 451 to 550 for the WT10G, respectively (see Table 2 ).

Table 2 lists the test TREC topics, the number of documents, and the standard deviation of document length in each collection. As shown in the last row, the document length distribution of the four collections is quite different, which indicates that the newly generated Dirichlet Priors-

\footnotetext{
${ }^{2}$ Related information of disk $1 \& 2$ and $\operatorname{disk} 4 \& 5$ of the TREC collections can be found from the following URL: http://trec.nist.gov/data/docs_eng.html
} 
Table 2: Details of the four TREC collections used in our experiments. The second row gives the number of topics associated to each collection. $N$ is the number of documents in the given collection. $\sigma_{l c o l l}$ is the standard deviation of document length in the whole collection.

\begin{tabular}{|c|l|l|l|l|}
\hline & disk1\&2 & disk4\&5 & WT2G & WT10G \\
\hline Topics & $51-200$ & $\begin{array}{l}301-450 \\
\text { and } \\
601-700\end{array}$ & $401-450$ & $451-550$ \\
\hline $\mathrm{N}$ & 741860 & 528155 & 247491 & 1692044 \\
\hline$\sigma_{\text {lcoll }}$ & 862.4977 & 558.1173 & 2009.3760 & 2303.4063 \\
\hline
\end{tabular}

based models and the tuning method are evaluated on diverse collections.

Each TREC topic consists of three fields, i.e. title, description and narrative. In this paper, we experiment with three types of queries with respect to the use of different topic fields, in order to check the impact of query length on the effectiveness of our models and the tuning method. The three types of queries are:

- Short queries: Only the title field is used.

- Normal queries: Only the description field is used.

- Long queries: All the three fields (title, description and narrative) are used.

Regarding the parameter setting of the baseline models, for the BM25 model, we use the default setting, which is $b=0.75, k_{1}=1.2$ and $k_{3}=1000$ [10]; For the PL2 model, we use the default setting applied in [1], which is $c=1$ for short queries and $c=7$ for long queries. Since [1] does not report experiments using normal queries, we use the optimal parameter setting on the disk $1 \& 2$ as the baseline, i.e. $c=1.4$ for normal queries; For the $t f \cdot i d f$ model, we also apply the default setting that is $k_{1}=1.2$ and $b=0.75$ [10].

For the Dirichlet Priors-based models, including BM3 and PL3, we test a series of values for the parameter $\mu$, ranging from 0 (exclude 0 ) to 10,000 , in order to extensively study the performance of the Dirichlet Priors-based models and show the need for the parameter tuning of the Dirichlet Priors normalisation.

Moreover, for testing our automatic tuning approach to the Dirichlet Priors normalisation, we use the disk1\&2 as the training collection, and evaluate on the other three collections. An advantage of using this training collection is that it has a relatively large number of available training queries, which are the TREC topics numbered from 51 to 200. After obtaining the optimal $\operatorname{corr}(t f n, l)$ value on the training collection using the corresponding relevance assessment, we evaluate our approach on the other three TREC collections. For the computation of the $\operatorname{corr}(t f n, l)$ value, terms that appear in only one document are ignored in order to avoid a zero correlation.

Our baseline for the evaluation of the tuning method is the optimal setting on the training collection, which is an empirical setting. Moreover, we compare the performance of our tuning method with the best manually obtained parameter setting using relevance assessment.

In all our experiments, standard stopwords removal and the Porter's stemming algorithm are applied. We used one AMD Athlon 1600 processor, running at $1.4 \mathrm{GHz}$.
Figure 1: The mean average precision (MAP) against the parameter $\mu$ for short queries on the four used TREC collections.

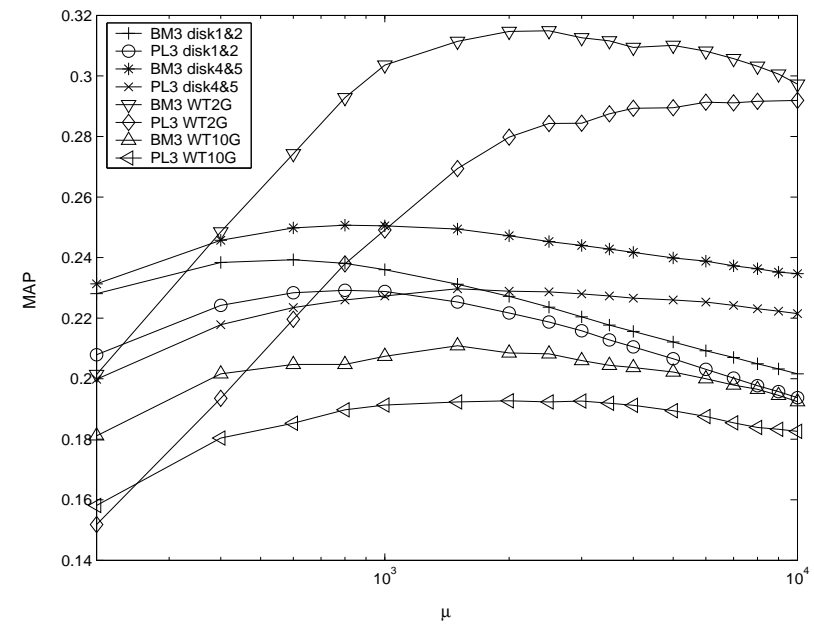

Table 3: The best manually obtained settings of the parameter $\mu$, selected from a wide range of values, on four collections for three types of queries.

\begin{tabular}{|c|c|c|c|}
\hline & Short & Normal & Long \\
\hline \multicolumn{4}{|c|}{ BM3 } \\
\hline disk1\&2 & 400 & 200 & 200 \\
disk4\&5 & 950 & 450 & 300 \\
WT2G & 2700 & 1400 & 650 \\
WT10G & 1500 & 400 & 500 \\
\hline \multicolumn{4}{|c|}{ PL3 } \\
\hline disk1\&2 & 800 & 200 & 200 \\
disk4\&5 & 1600 & 400 & 400 \\
WT2G & 9700 & 2300 & 1200 \\
WT10G & 2600 & 600 & 900 \\
\hline
\end{tabular}

\section{DISCUSSION OF RESULTS}

In Section 6.1, we start with presenting the results obtained using the Dirichlet Priors-based models with various parameter settings, showing the importance of our parameter tuning method. Then, we compare the performance of the Dirichlet Priors-based models with three baseline models. In Section 6.2, we show that our automatic tuning method achieves robust and effective performance. In particular, the tuning method's performance differs marginally from the best manually obtained setting using relevance assessment.

\subsection{Performance of the Dirichlet Priors-based Models}

Table 3 contains the manually obtained optimal parameter values on different collections with respect to the three query types. As we can see, on diverse collections, the optimal parameter values are quite different. Moreover, Figures 1, 2 and 3 sketch the plots of the parameter $\mu$ against mean average precision on the four used TREC collections for the three types of queries, respectively. In the figures, the curves of the parameter $\mu$ against mean average precision behave differently. Table 3 and Figures 1, 2 and 3 show that there is a justifiable need for the parameter tuning. 
Figure 2: The mean average precision (MAP) against the parameter $\mu$ for normal queries on the four used TREC collections.

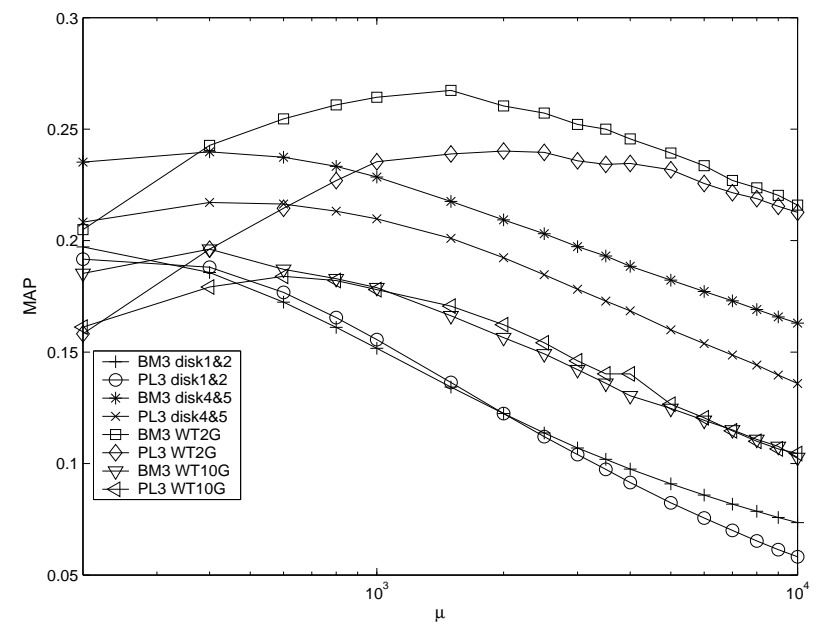

Figure 3: The mean average precision (MAP) against the parameter $\mu$ for long queries on the four used TREC collections.

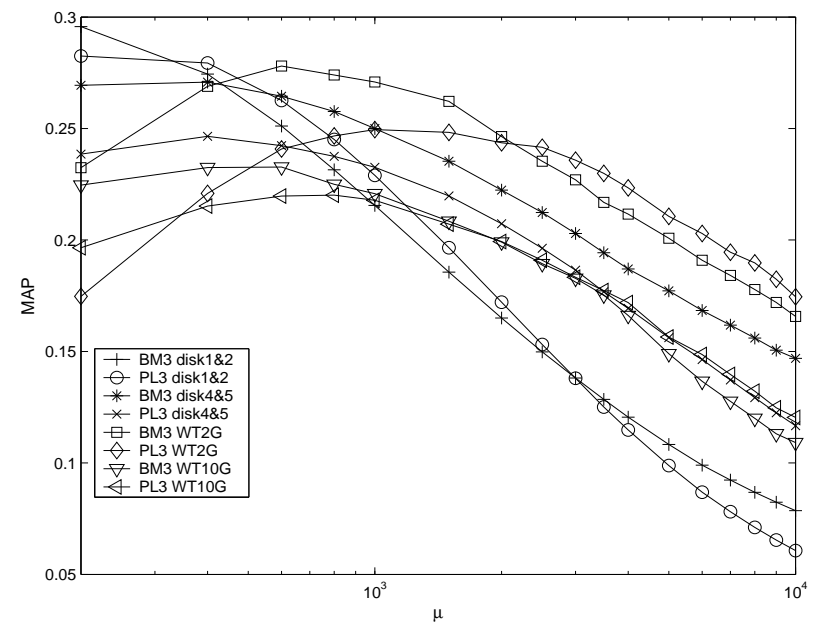

Table 4 provides the mean average precision obtained by using the baselines and the Dirichlet Priors-based models. From the table, we have the following observations:

- BM3 clearly outperforms BM25 for short queries, and achieves comparable performance for normal and long queries.

- Overall, PL2 provides slightly higher mean average precision than PL3, but PL3's performance is still shown to be robust and better than PL2 in some cases.

- The Dirichlet Priors-based models, i.e. BM3 and PL3, generally outperform $t f \cdot i d f$ for short and normal queries, and provide comparable performance for long queries.

Overall, as shown by the results, the newly generated Dirichlet Priors-based models outperform the $t f \cdot i d f$ model and achieve comparable performance with the robust BM25 and PL2 models.
Table 4: The obtained mean average precision on the four collections using the five models. The applied parameter settings for BM3 and PL3 are taken from Table 3 .

\begin{tabular}{|c|ccc|cc|}
\hline \multicolumn{5}{|c|}{ Short queries } \\
\hline disk1\&2 & .2214 & .2226 & .2338 & .2395 & .2293 \\
disk4\&5 & .2431 & .2418 & .2570 & .2508 & .2301 \\
WT2G & .2615 & .2600 & .3102 & .3157 & .2930 \\
WT10G & .1866 & .1868 & .2092 & .2109 & .1933 \\
\hline \multicolumn{7}{|c|}{ Normal queries } \\
\hline disk1\&2 & .1772 & .1913 & .1905 & .1972 & .1972 \\
disk4\&5 & .2437 & .2461 & .2366 & .2399 & .2171 \\
WT2G & .2407 & .2528 & .2407 & .2679 & .2410 \\
WT10G & .1739 & .1776 & .1779 & .1962 & .1840 \\
\hline \multicolumn{7}{|c|}{ Long queries } \\
\hline disk1\&2 & .2898 & .2981 & .2958 & .2957 & .2854 \\
disk4\&5 & .2797 & .2858 & .2704 & .2724 & .2465 \\
WT2G & .2772 & .2807 & .2520 & .2790 & .2523 \\
WT10G & .2290 & .2310 & .2235 & .2338 & .2220 \\
\hline
\end{tabular}

Table 5: The optimal parameter values and the corresponding correlation measures of the normalised $t f$ with the document length on the training collection. The value marked with $*$ is taken as the optimal constant $\operatorname{corr}(t f n, l)$ of our tuning method.

\begin{tabular}{|c|c|c|c|}
\hline & Short & Normal & Long \\
\hline & \multicolumn{3}{|c|}{ BM3 } \\
\hline$\mu$ & 400 & 200 & 200 \\
$\operatorname{corr}(t f n, l)$ & $-.1042^{*}$ & -.1086 & -.1253 \\
\hline & \multicolumn{3}{|c|}{ PL3 } \\
\hline$\mu$ & 800 & 200 & 200 \\
$\operatorname{corr}(t f n, l)$ & -.08581 & -.1086 & -.1253 \\
\hline
\end{tabular}

\subsection{Performance of the Automatic Tuning Ap- proach}

In this section, we start with presenting how we detect the optimal corr $(t f n, l)$ on the training collection ${ }^{3}$, and then discuss the obtained evaluation results.

Table 5 contains the optimal parameter values and corresponding $\operatorname{corr}(t f n, l)$, i.e correlation of the normalised term frequency with the document length on the training collection. Note that the provided correlation values are the mean of the correlation for each query term. We can see that the optimal parameter values for the two Dirichlet Priorsbased models are very similar, and for different types of queries, the optimal parameter settings result in relatively similar correlation measures. Although for short queries, the optimal settings are not identical $(\mu=800$ for BM3 and $\mu=400$ for PL3), for both models, $\mu=800$ and $\mu=400$ differs marginally from each other in terms of mean average precision ${ }^{4}$, indicating that the underlying hypothesis of our tuning method stands (see Section 4 for the hypothe-

\footnotetext{
${ }^{3}$ The notion of the "optimal corr $(t f n, l)$ " refers to the $\operatorname{corr}(t f n, l)$ value given by the optimal parameter setting. See step 1 of our tuning method in Section 4.

${ }^{4}$ For BM3, $\mu=400$ and $\mu=800$ provide mean average precision of .2384 and .2381 , respectively. For PL3, $\mu=400$ and $\mu=800$ provide mean average precision of .2242 and .2292 , respectively.
} 
Table 6: The correlation measures for some query terms on the training collection for different parameter values.

\begin{tabular}{|c|c|c|c|c|c|}
\hline$\mu$ & 200 & 600 & 1000 & 4000 & 8000 \\
\hline airbus & -.1307 & -.0752 & -.0487 & .0073 & .0256 \\
sanction & -.1184 & -.1069 & -.0963 & -.0470 & -.0157 \\
contract & -.0863 & -.0418 & -.0129 & .0742 & .1144 \\
merit & -.2231 & -.2164 & -.2026 & -.1367 & -.0959 \\
iran & -.0456 & -.0341 & -.0291 & -.0207 & -.0203 \\
\hline
\end{tabular}

sis). Therefore, for the tuning process, we take the optimal $\operatorname{corr}(t f n, l)$ for short queries using BM3 as the optimal constant $\operatorname{corr}(t f n, l)$ (see the value marked with a star in Table 3$)$. We choose the optimal $\operatorname{corr}(t f n, l)$ value for the short queries as the optimal constant correlation because of the fact that query terms in the titles are generally more informative than those in the descriptions and narratives. Therefore, terms in the titles can be more reliable than terms in other topic fields in inferring an optimal parameter setting. On a collection other than the training collection, we apply such a parameter setting that it gives this constant.

Table 6 presents some examples of the correlation measures on the training collection. As we can see, with respect to a particular parameter value, the correlation measures of different terms are diverse. Therefore, a term-based tuning approach might achieve higher precision/recall than just computing the mean of the correlation measures of query terms. However, as it is quite time-consuming to carry out a tuning process for each query term, we rather follow the proposed approach in this paper (see Section 4). Later we show that our approach achieves robust retrieval performance in the evaluation.

Tables 7 and 8 compare the mean average precision (MAP) obtained by using our tuning method with the MAP obtained by using the optimal values on the training collection. In the two tables, $M A P_{b}$ and $M A P_{t}$ stands for the mean average precision (MAP) obtained by using the baseline setting and the tuning method, respectively. $\mu$ stands for the parameter setting estimated by the tuning method. $\Delta$ is the percentage of improvement using the tuning method. A p-value marked with star indicates a significant difference between the results at 0.05 level according to the Wilcoxon test. As we can see, in most cases, our tuning method either significantly outperforms the baseline, or achieves comparable performance with the baseline.

Moreover, Tables 9 and 10 compare the performance of the tuning method with that of the manually optimal parameter setting obtained using relevance assessment. The notations in the two tables are the same as in Table 7. As can be seen from the tables, the performance of our tuning method is similar with the manual setting in most cases, and the difference of mean average precision is usually marginal. This indicates that the underlying hypothesis of our tuning method indeed holds (see Section 4 for the hypothesis). Overall, our tuning method provides effective and reliable retrieval performance over diverse TREC document and Web collections.

\section{CONCLUSION AND FUTUREDIRECTION}

In this paper, we have studied the application of the Dirichlet Priors to the term frequency normalisation. In particu-
Table 7: Results for BM3. This table compares the performance obtained by using the optimal setting on the training collection with that using the tuning method. The settings for $\mu$ are automatically obtained using our tuning method.

\begin{tabular}{|c|c|c|c|c|c|}
\hline \multicolumn{6}{|c|}{ Short query } \\
\hline disk4\&5 & 668 & .2490 & .2499 & +0.36 & $.02569^{*}$ \\
WT2G & 2266 & .2692 & .3151 & +17.05 & $1.174 \mathrm{e}-05^{*}$ \\
WT10G & 1782 & .2040 & .2093 & +2.60 & .3253 \\
\hline \multicolumn{6}{|c|}{ Normal query } \\
\hline disk4\&5 & 578 & .2352 & .2377 & +1.06 & .7677 \\
WT2G & 1514 & .2427 & .2674 & +10.18 & .08932 \\
WT10G & 1168 & .1853 & .1745 & -5.83 & .7098 \\
\hline \multicolumn{7}{|c|}{ Long query } \\
\hline disk4\&5 & 610 & .2694 & .2646 & -1.78 & .1841 \\
WT2G & 1441 & .2324 & .2624 & +4.30 & .1503 \\
WT10G & 1212 & .2246 & .2155 & -4.05 & .6677 \\
\hline
\end{tabular}

Table 8: Results for PL3. This table compares the performance obtained by using the optimal setting on the training collection with that using the tuning method. The settings for $\mu$ are automatically obtained using our tuning method.

\begin{tabular}{|c|c|c|c|c|c|}
\hline \multicolumn{6}{|c|}{ Short query } \\
\hline disk4\&5 & 668 & .2260 & .2243 & -0.75 & .0887 \\
WT2G & 2266 & .1935 & .2833 & +46.41 & $3.828 \mathrm{e}-07^{*}$ \\
WT10G & 1782 & .1804 & .1923 & +6.60 & $.002791^{*}$ \\
\hline \multicolumn{6}{|c|}{ Normal query } \\
\hline disk4\&5 & 578 & .2083 & .2168 & +4.08 & .00288 \\
WT2G & 1514 & .1582 & .2388 & +50.95 & $1.769 \mathrm{e}-06$ \\
WT10G & 1168 & .1792 & .1746 & -2.57 & .9238 \\
\hline \multicolumn{6}{|c|}{ Long query } \\
\hline disk4\&5 & 610 & .2385 & .2421 & +1.51 & .1378 \\
WT2G & 1441 & .1747 & .2495 & +42.82 & $2.461 \mathrm{e}-05$ \\
WT10G & 1212 & .2152 & .2135 & -0.79 & .8997 \\
\hline
\end{tabular}

lar, we have applied the Dirichlet Priors normalisation to a representative of two families of weighting models, i.e. the classical BM25 probabilistic model and the Divergence from Randomness PL2 model. By replacing the $t f$ normalisation components of the two models with the Dirichlet Priors normalisation, the newly generated weighting models are shown to be robust and effective in our experiments.

A major contribution of this paper is the proposed novel theoretically-driven automatic tuning method for the Dirichlet Priors normalisation. The proposed approach interprets the dependence between the normalised term frequency and the document length as the correlation between the two variables. Experiments on the TREC collections show that the underlying hypothesis of our tuning approach holds. Evaluation results also show that the tuning method significantly outperforms the baseline and its performance differs marginally from the manual setting using relevance assessment.

There are some interesting future directions that will help in better understanding the $t f$ normalisation. We plan to study the application of other smoothing methods, e.g. the Jelinek-Mercer smoothing $[3,8]$, to the $t f$ normalisation. 
Table 9: Results for BM3. This table compares the performance obtained manually using relevance assessment with that using the automatic tuning method.

\begin{tabular}{|c|c|c|c|c|}
\hline & $M A P_{b}$ & $M A P_{t}$ & $\Delta$ & p-value \\
\hline \multicolumn{5}{|c|}{ Short query } \\
\hline disk4\&5 & .2508 & .2499 & -0.36 & .07424 \\
WT2G & .3157 & .3151 & -0.19 & .7196 \\
WT10G & .2109 & .2093 & -0.76 & .3567 \\
\hline \multicolumn{5}{|c|}{ ormal query } \\
\hline disk4\&5 & .2399 & .2377 & -0.92 & $3.709 \mathrm{e}-4^{*}$ \\
WT2G & .2679 & .2674 & -0.19 & .1812 \\
WT10G & .1962 & .1745 & -11.06 & $.006093^{*}$ \\
\hline \multicolumn{5}{|c|}{ Long query } \\
\hline disk4\&5 & .2724 & .2646 & -2.86 & $6.769 \mathrm{e}-4^{*}$ \\
WT2G & .2790 & .2624 & -5.95 & $.0245^{*}$ \\
WT10G & .2338 & .2155 & -7.83 & $.002763^{*}$ \\
\hline
\end{tabular}

Table 10: Results for PL3. This table compares the performance obtained manually using relevance assessment with that using the automatic tuning method.

\begin{tabular}{|c|c|c|c|c|}
\hline & $M A P_{b}$ & $M A P_{t}$ & $\Delta$ & p-value \\
\hline \multicolumn{5}{|c|}{ Short query } \\
\hline disk4\&5 & .2271 & .2243 & -1.23 & $6.137 \mathrm{e}-07^{*}$ \\
WT2G & .2930 & .2833 & -3.31 & .2732 \\
WT10G & .1933 & .1923 & -0.52 & .8031 \\
\hline \multicolumn{5}{|c|}{ Normal query } \\
\hline disk4\&5 & .2169 & .2168 & $\approx 0$ & .2573 \\
WT2G & .2410 & .2388 & -0.91 & .6816 \\
WT10G & .1840 & .1746 & -5.22 & $.03436^{*}$ \\
\hline \multicolumn{5}{|c|}{ Long query } \\
\hline disk4\&5 & .2465 & .2421 & -1.78 & $.005519^{*}$ \\
WT2G & .2523 & .2495 & -1.11 & .2127 \\
WT10G & .2220 & .2135 & -3.83 & $.0175^{*}$ \\
\hline
\end{tabular}

In particular, we will apply the proposed tuning method to these classical smoothing methods.

It will also be interesting to device a term-based tuning mechanism for the Dirichlet Priors normalisation. As suggested previously, a term-based tuning mechanism could achieve a better retrieval performance though it would have a high computational cost. A possible solution for lowering the overhead is to enable tuning only for the most informative terms in a query, while applying the default parameter setting for the rest of the query terms.

\section{ACKNOWLEDGMENTS}

This work is funded by the Leverhulme Trust, grant number F/00179/S. The project funds the development of the Smooth project, which investigates the term frequency normalisation (URL: http://ir.dcs.gla.ac.uk/smooth).

The experiments were conducted using Terrier's IR platform, version 1.0.0. It is a modular platform for the rapid development of large-scale IR applications, providing indexing and retrieval functionalities. More information can be found from http://ir.dcs.gla.ac.uk/terrier.

\section{REFERENCES}

[1] G. Amati. Probabilistic Models for Information Retrieval based on Divergence from Randomness. PhD thesis, Department of Computing Science, University of Glasgow, 2003.

[2] G. Amati and C. J. van Rijsbergen. Probabilistic models of Information Retrieval based on measuring the divergence from randomness. In $A C M$

Transactions on Information Systems (TOIS), volume 20(4), pages 357 - 389, October 2002.

[3] S. Chen and J. Goodman. An empirical study of smoothing techniques for language modeling. In Proceedings of the Thirty-Fourth Annual Meeting of the Association for Computational Linguistics, pages 310 - 318, San Francisco, CA, 1996.

[4] M. DeGroot. Probability and Statistics. Addison Wesley, 2nd edition edition, 1989.

[5] D. Hawking. Overview of the TREC-9 Web Track. In Proceedings of the Nineth Text REtrieval Conference (TREC-9), pages 87 - 94, Gaithersburg, MD, 2000.

[6] D. Hawking, E. Voorhees, N. Craswell, and P. Bailey. Overview of the TREC- 8 Web Track. In Proceedings of the Eighth Text REtrieval Conference (TREC-8), pages 131 - 150, Gaithersburg, MD, 1999.

[7] B. He and I. Ounis. Tuning Term Frequency Normalisation for BM25 and DFR Models. In Proceedings of the 27th European Conference on Information Retrieval (ECIR'05), pages $200-214$, Santiago de Compostela, Spain, March, 2005.

[8] F. Jelinek and R. Mercer. Interpolated estimation of markov source parameters from sparse data. In E. S. Gelsema and L. N. Kanal, editors, Pattern Recognition in Practice, pages 381 - 402, Amsterdam, The Netherlands, 1980.

[9] C. J. van Rijsbergen. Information Retrieval, 2nd edition. Department of Computer Science, University of Glasgow, 1979.

[10] S. Robertson, S. Walker, M. Beaulieu, M. Gatford, and A. Payne. Okapi at TREC-4. In NIST Special Publication 500-236: The Fourth Text REtrieval Conference (TREC-4), pages 73 - 96, Gaithersburg, MD, 1995.

[11] A. Singhal, C. Buckley, and M. Mitra. Pivoted document length normalization. In Proceedings of the 19th Annual International ACM SIGIR Conference on Research and Development in Information Retrieval, pages 21 - 29, Zurich, Switzerland, 1996.

[12] K. Sparck-Jones. A statistical interpretation of term specificity and its application to retrieval. Journal of Documentation, (28):11 - 21, 1972.

[13] C. Zhai and J. Lafferty. A study of smoothing methods for language models applied to ad hoc Information Retrieval. In Proceedings of the 24th Annual International ACM SIGIR Conference on Research and Development in Information Retrieval, pages 334 - 342, New Orleans, LA, 2001. 